\title{
Erratum to: The effects of arginase inhibitor on lung oxidative stress and inflammation caused by pneumoperitoneum in rats
}

\author{
Jin Sun Cho', Young Jun Oh ${ }^{1,2}$, Ok Soo Kim² and Sungwon $\mathrm{Na}^{1,2^{*}}$
}

\section{Erratum}

In this version of this article that was originally published [1] there is an error in the Acknowledgements. Where it says 'This study was supported by a faculty research grant of Yonsei University College of Medicine (6-2010-0163), the number should be 6-2012-0012, not 6-2010-0163.

Received: 28 November 2016 Accepted: 28 November 2016

Published online: 13 December 2016

\section{Reference}

1. Cho JS, Oh YJ, Kim OS, Na S. The effects of arginase inhibitor on lung oxidative stress and inflammation caused by pneumoperitoneum in rats. BMC Anesthesiol. 2016;15:129.

\footnotetext{
* Correspondence: NSWKSJ@yuhs.ac

${ }^{1}$ Department of Anesthesiology and Pain Medicine, Yonsei University College of Medicine, Seoul, Republic of Korea

2Department of Anesthesiology and Pain Medicine, Anesthesia and Pain Research Institute, Yonsei University College of Medic, Seoul, Republic of Korea
} 